\title{
Thermal Stability and Luminescent Properties of Tri-cellulose Acetate Composites Containing Dy $(\mathrm{SSA})_{3}$ Phen Complex
}

\author{
Xiangwei Sun, Feiyue Wu, Yan Luo, Mengjun Huang (D, Yuntao Li, Xiang Liu, \\ and Kunpeng Liu \\ College of Materials and Chemical Engineering, Chongqing University of Arts and Sciences, Honghe Road 319, \\ Chongqing 402160, China \\ Correspondence should be addressed to Mengjun Huang; huangmj806@gmail.com
}

Received 8 May 2018; Revised 29 July 2018; Accepted 3 September 2018; Published 24 September 2018

Academic Editor: Marco Anni

Copyright ( 2018 Xiangwei Sun et al. This is an open access article distributed under the Creative Commons Attribution License, which permits unrestricted use, distribution, and reproduction in any medium, provided the original work is properly cited.

Dysprosium (Dy) ternary complex was prepared using 5-sulfosalicylic acid (SSA) as the first ligand and 1,10-phenanthroline (Phen) as the second ligand, denoted as Dy(SSA) ${ }_{3}$ Phen. The complex was blended with tri-cellulose acetate (TCA) via a cosolvent method to obtain polymer luminescent materials. The composition and structure of the rare-earth complex were characterized by means of elemental analysis, infrared (IR) spectra, and thermogravimetric analysis (TGA). The fluorescence spectra displayed this pure $\mathrm{Dy}(\mathrm{SSA})_{3}$ Phen complex, and the TCA/Dy(SSA) ${ }_{3}$ Phen composites all emit blue light. The (90/10) composite possesses fine luminescent properties with quantum yield of $33.5 \%$ and thermal stability for potential usage as blue fluorescent materials.

\section{Introduction}

Polymer/rare earth (RE) composites possess excellent optical, electrical, and spectroscopic properties stemming from the additive rare-earth compounds and meantime have fine processability and flexibility arising from polymer materials, which can be potentially applied in light-emitting diodes, detector systems, X-ray phosphors, solid-state lasers and bioinorganic sensors, etc. [1-5]. Considerable attention has been paid to design and synthesize polymer/RE luminescent materials with good luminescent properties and high internal quantum efficiencies [6-8]. RE-based emitters which are usually doped or bonded with macromolecules can generate characteristically sharp and narrow emissions, because of the distinctive excitation mechanism of antenna effect and $\mathrm{f}-\mathrm{f}$ radiative transition [9-14]. A considerable number of research reports have focused on four kinds of RE metals, i.e., europium (Eu), terbium (Tb), samarium $(\mathrm{Sm})$, and dysprosium (Dy), especially for the Eu or Tb ions based complexes, because their resonance energy levels are close to the triplet levels of organic ligands, and so the RE ions could emit strong luminescent properties via the efficient energy transfer between ligands and RE ions [15-19].
Notably, doping luminescent RE organic complexes into polymer matrices is one of the most effective approaches to obtain the polymer luminescent materials via a simple, convenient, and high efficient blending method. However, some doped-polymer/RE composites exhibited poor luminescent properties and poor mechanical properties, mainly owing to the nonuniform dispersion of RE complexes in polymer matrices and the poor chemical affinity between the doped complex and polymer matrix $[9,20]$. In order to solve these problems, organic ligands of RE complexes play a significant role in boosting the chemical affinity between the added complexes and macromolecules, and especially the organic compounds possessing multifunctional groups which could be potential hydrogen-bonding acceptors or donors are primarily selected as ligands for RE complexes [21-23]. Conjugated acids were usually used as the first ligand as a result of their strong coordination ability with RE ions and the potential hydrogen-bonding acceptors from carbonyl groups, e.g., salicylic acid [13], phthalic acid [24], benzoic acid and its derivatives [25], and acrylic acid [26]. It is worthwhile pointing out that the molecular structure of polymer matrices is another significant factor for 
promoting the chemical affinity between the added RE complexes and polymer chains.

In this paper, tri-cellulose acetate (TCA) was selected as the polymer matrix, because of the potential hydrogenbonding donors from the substantial hydroxyl groups. Furthermore, as a result of good filming performance, excellent transparency, fine glossiness, well solubility, and good processability of TCA, it had been widely used in the protective film of the polarizing film of the liquid-crystal display (LCD) which required a high transparency [27]. TCA could be easily soluble in a variety of solvents, favorable to prepare polymer/RE composites by means of solution blending. Additionally, sulfosalicylic acid (SSA) was chosen as the first ligand, not only because it could sensitize the luminescent property of Dy ions but also since its oxygen of carbonyl groups provides hydrogen-bonding acceptors $[13,19]$. Therefore, the hydrogen bonding is most probably formed between the ligands of the added RE complex and the TCA matrix, favorable to improve the uniform dispersion of the complex in the matrix. As a series of studies on polymer luminescent materials emitting three-color light (red, green, and blue) [10, 28], Dy-based complexes blended with TCA have been highly concerned. There are few reports of $\mathrm{Dy}^{3+}$ complex-doped polymer materials with fine luminescence, and thus, we synthesized $\mathrm{Dy}^{3+}$ ternary complexes using SSA and 1,10-phenanthroline (Phen) as the first and second ligands, respectively. The $\mathrm{Dy}(\mathrm{SSA})_{3}$ Phen complex can retain fine luminescent properties dispersing into the TCA matrix, and finally, the TCA/Dy(SSA) ${ }_{3}$ Phen composites emitting blue light with good thermal stability were obtained.

\section{Materials and Methods}

2.1. Materials. Tri-cellulose acetate (TCA), with the weightaverage molecular weight of 200,000, was supplied by our collaborating laboratory (Xiuli Wang's Research Group, Sichuan University) and purified by acetone. Dysprosium oxide $\left(\mathrm{Dy}_{2} \mathrm{O}_{3}\right)$ at $99.99 \%$ purity was provided by Shanghai Yuelong Rare Earth New Materials Co., Ltd., China. 5sulfosalicylic acid (SSA, AR), 1,10-phenanthroline (Phen, AR), dimethyl sulfoxide (DMSO, AR), acetone (EA, AR), and absolute ethyl alcohol (AR) were purchased from Chengdu Kelong Chemical Reagent Factory, China. Sodium hydroxide $(\mathrm{NaOH}, \mathrm{AR})$ was purchased from Chongqing Chuandong Chemical Co., Ltd., China. Concentrated hydrochloric acid ( $\mathrm{HCl}, \mathrm{AR})$ was supplied by Chongqing Inorganic Chemical Reagent Factory, China.

\subsection{Preparation of $D y(S S A)_{3}$ Phen Complex. Weighed} $3 \mathrm{mmol} \mathrm{Dy} \mathrm{O}_{3}$ and $80 \mathrm{ml}$ concentrated hydrochloric acid in a $100 \mathrm{ml}$ bottom beaker immersed in an oil bath with magnetic stirrer, heated to $100^{\circ} \mathrm{C}$ to dissolve $\mathrm{Dy}_{2} \mathrm{O}_{3}$ powders absolutely, kept at the temperature for 10 hours to evaporate the solution to dryness and then cooled to room temperature. Then dissolved the remains using absolute EA in a $250 \mathrm{ml}$ volumetric flask, added absolute EA to the mark, and finally got $0.1 \mathrm{~mol} / \mathrm{L} \mathrm{DyCl}_{3}$ ethanol solution. About
$15 \mathrm{mmol}$ SSA, $5 \mathrm{mmol}$ Phen, and $30 \mathrm{ml}$ absolute EA were successively added in $100 \mathrm{ml}$ beaker immersed in an oil bath with a magnetic stirrer to dissolve all components absolutely, and then, $5 \mathrm{mmol} \mathrm{Dy}^{3+}$ ethanol solution was added in dropwise, keeping the reaction temperature at $80^{\circ} \mathrm{C}$. The $\mathrm{pH}$ value of the mixture was adjusted to about 6.5 with $\mathrm{NaOH}$ aqueous solution $(6 \mathrm{~mol} / \mathrm{L})$, and the mixed solution was kept at $80^{\circ} \mathrm{C}$, stirred for 4 hours, and cooled to room temperature. The precipitates were repeatedly washed with EA and deionized water to remove chloride ions, dried in a vacuum oven at $60^{\circ} \mathrm{C}$ for 24 hours, and then stored in a damp-proof cabinet. The ternary complex, $\mathrm{Dy}(\mathrm{SSA})_{3}$ Phen, was prepared.

2.3. Preparation of $\mathrm{TCA} / \mathrm{D} y(\mathrm{SSA})_{3}$ Phen Composites. Dissolved $0.50 \mathrm{~g}$ solid mixtures of the purified TCA and Dy $(\mathrm{SSA})_{3}$ Phen complex powders using $30.0 \mathrm{ml}$ DMSO in a $100 \mathrm{ml}$ flat bottom beaker immersed in an oil bath with stirring, heated the mixed solution to $60^{\circ} \mathrm{C}$ with stirring, then cooled to room temperature, and underwent a natural evaporation process until the solution became semisolid samples. The prepared semisolid samples were transferred to an evaporating dish with flat bottom and dried in a vacuum oven at $60^{\circ} \mathrm{C}$ for 10 hours. Finally thin film-like TCA/Dy $(\mathrm{SSA})_{3}$ Phen composites were obtained and stored in a damp-proof cabinet. The weight ratios of TCA to Dy $(\mathrm{SSA})_{3}$ Phen were $100 / 0,96 / 4,93 / 7,90 / 10$, and $87 / 13$.

2.4. Characterization. Elemental analysis of $\mathrm{C}, \mathrm{H}$, and $\mathrm{N}$ in the pure $\mathrm{Dy}(\mathrm{SSA})_{3}$ Phen complex was conducted on an Elemental analyzer (Vario EL III, Elementar Co., Germany).

Infrared transmittance spectrum was recorded on a Nicolet 6670 FTIR spectrophotometer (Thermo Fisher Corp., USA). Infrared attenuated total reflection (IRATR) was implemented and measured by averaging 32 scans at a resolution of $4 \mathrm{~cm}^{-1}$ over the range $4000-450 \mathrm{~cm}^{-1}$.

Thermogravimetric analysis (TGA) was implemented by a thermogravimeter (Q500, TA Instruments Co., USA) in the nitrogen atmosphere using a heating rate of $10^{\circ} \mathrm{C} / \mathrm{min}$ from room temperature to $1000^{\circ} \mathrm{C}$.

The luminescent properties of the pure $\mathrm{Dy}(\mathrm{SSA})_{3}$ Phen complex and all TCA/Dy(SSA) ${ }_{3}$ Phen composites were characterized by a fluorescence spectrophotometer (Hitachi F-7000, Hitachi High-Technologies Corp., Japan). The adopted mode was luminescence. The supplied PMT voltage and scan speed were set to $400 \mathrm{~V}$ and $1200 \mathrm{~nm} / \mathrm{min}$, respectively. The excitation (EX) and emission (EM) slit widths were fixed at 2.5 and $5.0 \mathrm{~nm}$.

\section{Results and Discussion}

3.1. Elemental Analysis. The results of elemental analysis for the $\mathrm{Dy}(\mathrm{SSA})_{3}$ Phen complex are, respectively, listed as follows: measured value (theoretical value) C $41.6 \%$ (40.7\%), H $2.3 \%(2.5 \%)$, N $4.8 \%$ (5.0\%), and S 6.5\% (5.7\%). These results suggest that the molar ratio of $\mathrm{Dy}^{3+}$ to SSA to Phen is $1: 3: 1$ in the prepared $\mathrm{Dy}(\mathrm{SSA})_{3}$ Phen complex. 
3.2. FTIR Analysis. Figure 1 shows infrared spectra of the two ligands and the Dy(SSA) ${ }_{3}$ Phen complex, in the wavelength range of 3550 to $450 \mathrm{~cm}^{-1}$. As for the spectrum of Phen, the peak at $1561 \mathrm{~cm}^{-1}$ is assigned to the stretching vibration of $-\mathrm{N}=\mathrm{C}$ group, and the peaks at $852 \mathrm{~cm}^{-1}$ and $739 \mathrm{~cm}^{-1}$ are ascribed to the out-of-plane bending vibration of $-\mathrm{C}-\mathrm{H}$ group, and for the spectrum of SSA, the peak at $1656 \mathrm{~cm}^{-1}$ belongs to the stretching vibration of the carbonyl group. Obviously, compared with the spectrum of Phen, the peak of the spectrum of the $\mathrm{Dy}(\mathrm{SSA})_{3}$ Phen complex corresponding to the $\nu_{\mathrm{N}=\mathrm{C}}$ vibration shifts from $1561 \mathrm{~cm}^{-1}$ to $1536 \mathrm{~cm}^{-1}$, and the peaks belonging to the $r_{\mathrm{C}-\mathrm{H}}$ vibration shift from $852 \mathrm{~cm}^{-1}$ and $739 \mathrm{~cm}^{-1}$ to $843 \mathrm{~cm}^{-1}$ and $719 \mathrm{~cm}^{-1}$, respectively, which indicates that there are coordination bonds between two nitrogen atoms from Phen ligand and the central ion $\left(\mathrm{Dy}^{3+}\right.$ ion) in the complex [29]. Moreover, by comparison with the spectrum of SSA ligand, the peak of the spectrum of the complex assigned to the $v_{\mathrm{C}=\mathrm{O}}$ vibration shifts from $1656 \mathrm{~cm}^{-1}$ to $1621 \mathrm{~cm}^{-1}$, indicative of the formation of the coordination bonds between carbonyl group from SSA ligand and $\mathrm{Dy}^{3+}$ ion $[13,30]$. Furthermore, it is worth noting that as for the spectrum of the complex two new absorption peaks appearing at $549 \mathrm{~cm}^{-1}$ and $461 \mathrm{~cm}^{-1}$ are attributed to the stretching vibration of $\mathrm{O} \longrightarrow \mathrm{RE}$ and $\mathrm{N} \longrightarrow \mathrm{RE}$, respectively [31].

Infrared spectra of TCA and the TCA/Dy(SSA) ${ }_{3}$ Phen (95/5) and (90/10) composites are displayed in Figure 2, ranging from $4000 \mathrm{~cm}^{-1}$ to $450 \mathrm{~cm}^{-1}$. It can be seen that the two peaks of the spectrum of TCA occurring at $3480 \mathrm{~cm}^{-1}$ and $1740 \mathrm{~cm}^{-1}$ are assigned to the $\nu_{\mathrm{O}-\mathrm{H}}$ and $\nu_{\mathrm{C}=\mathrm{O}}$ vibrations, respectively. Clearly, the spectrum of the (95/5) composite is similar to that of the pure TCA except that the peak corresponding to the $\nu_{\mathrm{O}-\mathrm{H}}$ vibration of the composite shifts from $3480 \mathrm{~cm}^{-1}$ to $3415 \mathrm{~cm}^{-1}$. The possible reason is that the amount of the added Dy(SSA) ${ }_{3}$ Phen complex is comparatively low so that the characteristic peaks of the complex are almost covered by the strong peaks of TCA matrix. Nevertheless, as for the spectrum of the (90/10) composite, the peaks at $3393 \mathrm{~cm}^{-1}$ and $1740 \mathrm{~cm}^{-1}$ are, respectively, attributed to the $\nu_{\mathrm{O}-\mathrm{H}}$ and $\nu_{\mathrm{C}=\mathrm{O}}$ vibrations of the TCA matrix, and the peak at $1625 \mathrm{~cm}^{-1}$ is assigned to the $\nu_{\mathrm{C}=\mathrm{O}}$ vibration of SSA from the added complex. Furthermore, the main characteristic peaks of Dy(SSA) ${ }_{3}$ Phen complex at $846 \mathrm{~cm}^{-1}$, $717 \mathrm{~cm}^{-1}$, and $470 \mathrm{~cm}^{-1}$ also appear in the spectrum of the (90/10) composite. Compared with the spectra of the pure TCA and Dy(SSA) ${ }_{3}$ Phen complex as shown in Figures 1 and 2 , it can be found that the $\nu_{\mathrm{O}-\mathrm{H}}$ peak for the $(90 / 10)$ composite is red-shifted about $90 \mathrm{~cm}^{-1}$ and the $v_{\mathrm{C}=\mathrm{O}}$ peak for the composite is red-shifted $4 \mathrm{~cm}^{-1}$ from the $1621 \mathrm{~cm}^{-1}$, indicative of the existence of hydrogen bonds between the oxygen atom from carbonyl groups from SSA and the hydroxyl groups from TCA.

3.3. Thermal Analysis. Thermal properties of pure Dy $(\mathrm{SSA})_{3}$ Phen complex and TCA/Dy(SSA) ${ }_{3}$ Phen composites were characterized via TG analysis, and the TGA curves are shown in Figure 3. As for the plot of the Dy(SSA) ${ }_{3}$ Phen complex, there is a two-stage decomposition process, corresponding to the removal processes of two ligands. The total mass loss of the complex is $79.2 \%$, which is in good agreement with the calculated value $(80.9 \%)$ when the ligands of the $\mathrm{Dy}(\mathrm{SSA})_{3}$ Phen complex are completely decomposed and the final residues are rare-earth oxide [32]. The weight loss of the first stage is $58.6 \%$ which is considered as the removal process of SSA ligand, and that of the second stage is $20.4 \%$ belonging to the decomposition process of Phen ligand. Furthermore, at the first decomposition stage of the complex, the extrapolated onset $\left(T_{\mathrm{e}}\right)$ and final $\left(T_{\mathrm{c}}\right)$ temperatures are about $273^{\circ} \mathrm{C}$ and $325^{\circ} \mathrm{C}$, and at the second stage, the $T_{\mathrm{e}}$ and $T_{\mathrm{c}}$ are about $557^{\circ} \mathrm{C}$ and $669^{\circ} \mathrm{C}$, suggesting that the $\mathrm{Dy}(\mathrm{SSA})_{3}$ Phen complex possesses fitting thermal stabilities for the usage of luminescent materials. Based on the above-mentioned elemental analysis, FTIR analysis, and TGA results, the molecular structure of the $\mathrm{Dy}(\mathrm{SSA})_{3} \mathrm{Phen}$ complex can be ascertained, as shown in Figure 4.

As for TCA and the TCA composites, the TGA thermograms are displayed in Figure 3, and the $T_{\mathrm{e}}$ and $T_{\mathrm{c}}$ values as well as the mass-loss values are listed in Table 1. Obviously, there is only one stage of decomposition occurring on the TGA curves of TCA and the composites, which suggests that the decomposition process of the composites is similar to that of the pure TCA, and during the processes, each chain scission reaction has an analogous energy barrier [33]. It can be seen that with the increase of the content of the added complex, the $T_{\mathrm{e}}, T_{\mathrm{i}}$, and $T_{\mathrm{c}}$ values show a descending trend. This is probably caused by the fact that the degradation process of TCA macromolecules is related to the rupture of the anhydroglucose ring, i.e., the backbone rupture as well as the carbonization process and the volatile substances released from organic ligands of the added complex are probably favorable to boost the rupture process of TCA macromolecular chains [34]. The more the additive content of the complex, the more the released substances and the faster the degradation processes of the TCA composites. However, it is particularly worth pointing out that the $T_{\mathrm{e}}$ and $T_{\mathrm{i}}$ values of all composites are nearly over $300^{\circ} \mathrm{C}$, much higher than the onset temperature. As a consequence, the TCA/Dy(SSA) ${ }_{3}$ Phen composites still possess practicable thermal properties for materials.

3.4. Photoluminescent Properties. Figures 5 and 6 show the excitation and emission spectra of pure $\mathrm{Dy}(\mathrm{SSA})_{3}$ Phen complex and all TCA/Dy(SSA) ${ }_{3}$ Phen composites, respectively. It is obvious that the profile of the spectra of all composites is similar to that of the pure complex, i.e., there are broad and strong bands ranging from $260 \mathrm{~nm}$ to $360 \mathrm{~nm}$ in all excitation spectra, which suggests that the pure complex and the TCA composites can be well excited in the given wavelength range, as shown in Figure 5. The maximal values of the excitation spectra are the optimum excitation wavelengths for emission spectra, i.e., $326 \mathrm{~nm}$ is the optimum excitation wavelength for the pure $\mathrm{Dy}(\mathrm{SSA})_{3}$ Phen complex; $309 \mathrm{~nm}, 309 \mathrm{~nm}, 312 \mathrm{~nm}$, and $312 \mathrm{~nm}$ are corresponding to the TCA/Dy(SSA) ${ }_{3}$ Phen $(96 / 4,93 / 7,90 / 10$, and 87/13) composites, respectively. Meanwhile, it can be seen that the excitation spectra of the composites represent 


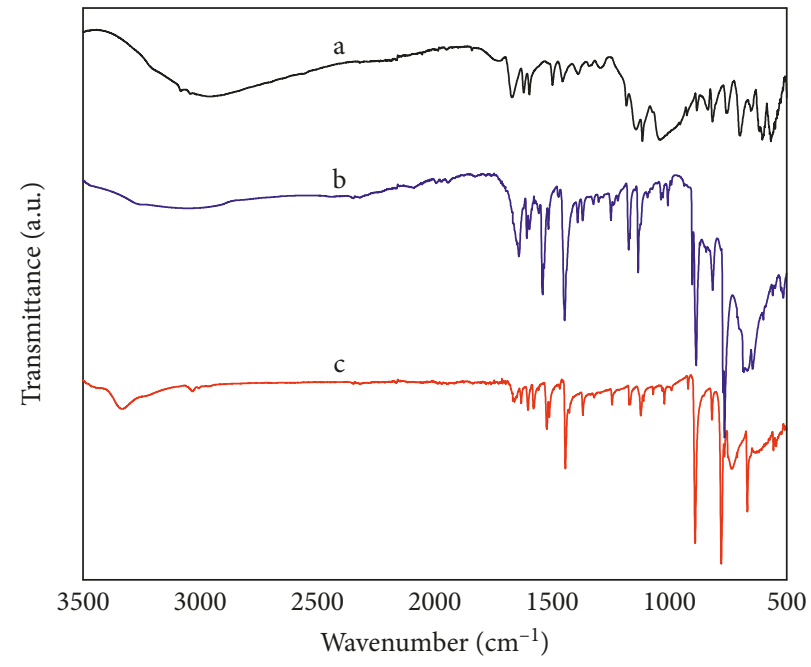

(a)

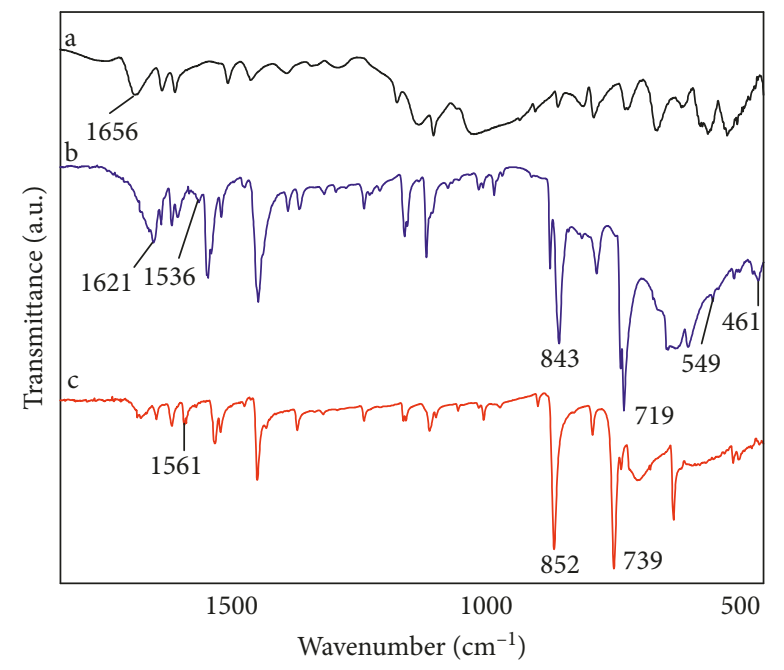

(b)

Figure 1: FTIR spectra ((a) $3550-450 \mathrm{~cm}^{-1}$; (b) $1800-450 \mathrm{~cm}^{-1}$ ) of ligands and the complex: a, SSA; b, Dy(SSA) ${ }_{3}$ Phen; c, Phen.

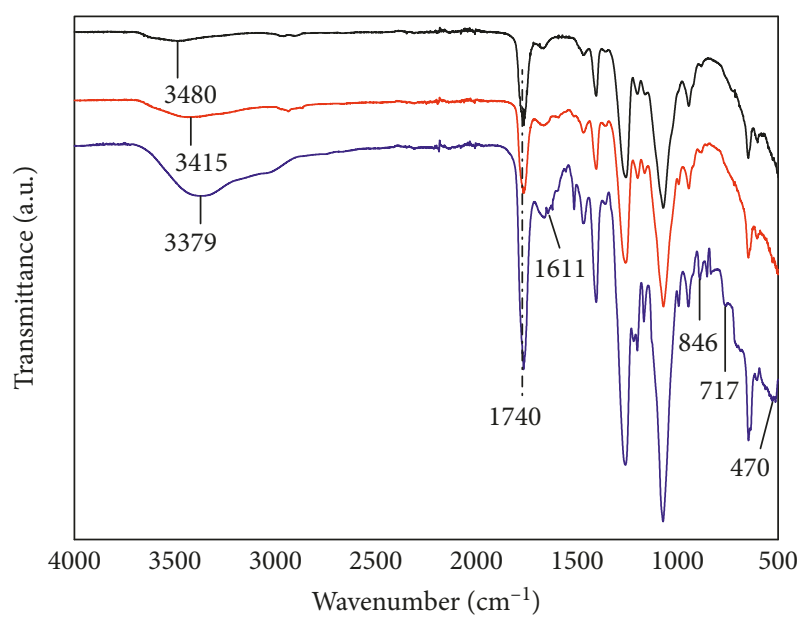

FIGURE 2: FTIR spectra of TCA and the TCA/Dy(SSA) ${ }_{3}$ Phen composites: a, TCA; b, (96/4); c, (90/10).

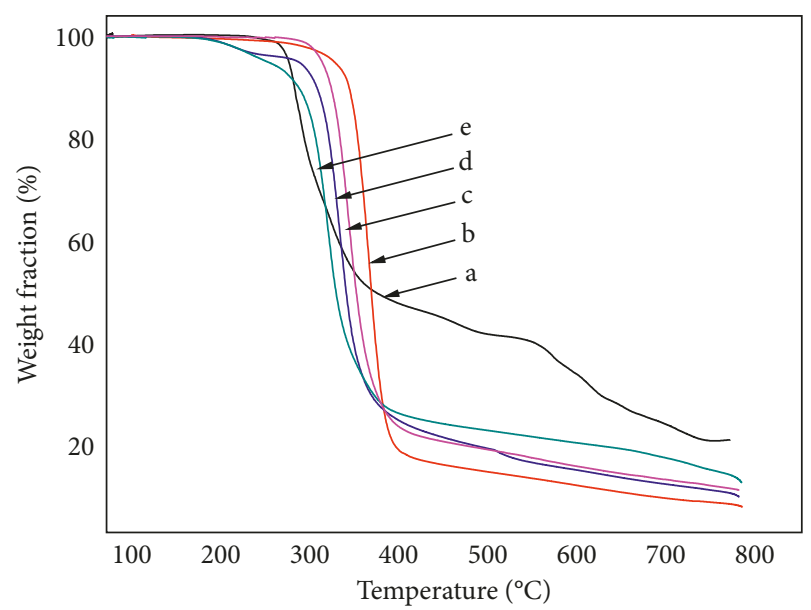

Figure 3: TGA thermograms of pure Dy(SSA $)_{3}$ Phen complex and TCA/Dy(SSA $)_{3}$ Phen composites: a, Dy(SSA) ${ }_{3}$ Phen; b, TCA; c, (96/4); d, (93/7); e, (90/10).

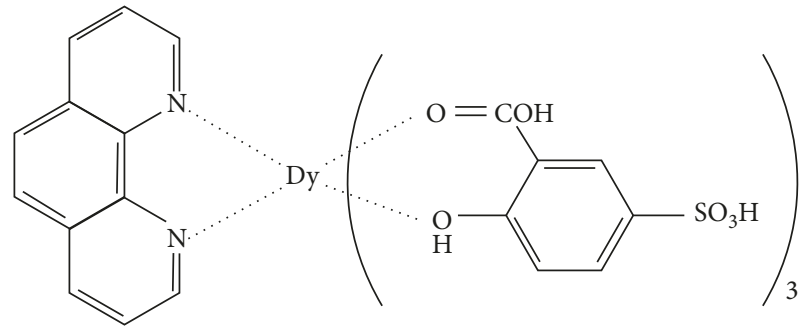

Figure 4: Molecular structure of the Dy(SSA) ${ }_{3}$ Phen complexes.

TABLe 1: The $T_{\mathrm{e}}, T_{\mathrm{i}}, T_{\mathrm{c}}$, and mass-loss values of pure TCA and the TCA/Dy(SSA $)_{3}$ Phen composites.

\begin{tabular}{lcccc}
\hline TCA/Dy(SSA $)_{3}$ Phen & $T_{\mathrm{e}}\left({ }^{\circ} \mathrm{C}\right)$ & $T_{\mathrm{i}}\left({ }^{\circ} \mathrm{C}\right)$ & $T_{\mathrm{c}}\left({ }^{\circ} \mathrm{C}\right)$ & Mass loss $(\%)$ \\
\hline$(100 / 0)$ & 347.0 & 366.9 & 380.1 & 92.1 \\
$(96 / 4)$ & 325.9 & 342.1 & 364.3 & 89.9 \\
$(93 / 7)$ & 313.4 & 331.3 & 355.2 & 88.6 \\
$(90 / 10)$ & 297.7 & 316.9 & 342.6 & 86.6 \\
\hline
\end{tabular}

$T_{\mathrm{e}}, T_{\mathrm{i}}$, and $T_{\mathrm{c}}$ denote extrapolated onset, onset, and final temperatures, respectively.

obvious blueshift compared with the $326 \mathrm{~nm}$ peak value for the pure complex, and especially for the (96/4) and (93/7) composites, the peaks are blueshifted by $17 \mathrm{~nm}$, indicating that TCA macromolecules may influence the excitation process of the added complex. The main reason is that the interaction between TCA and the added $\mathrm{Dy}(\mathrm{SSA})_{3}$ Phen complex as discussed in FTIR analysis probably affects the energy transfers of the SSA ligands during the excitation process of the doped complex in TCA composites.

Emission spectra of TCA/Dy(SSA) ${ }_{3}$ Phen $(0 / 100,96 / 4$, $93 / 7,90 / 10$, and $87 / 13$ ) composites were obtained by exciting at $326 \mathrm{~nm}, 312 \mathrm{~nm}, 312 \mathrm{~nm}, 309 \mathrm{~nm}$, and $309 \mathrm{~nm}$, respectively, as shown in Figure 6. For the pure Dy (SSA) ${ }_{3}$ Phen complex, the strong emission band with a maximum at $436 \mathrm{~nm}$ is assigned to $\pi \longrightarrow \pi^{*}$ electron transition of the SSA ligands and the weak band at around 


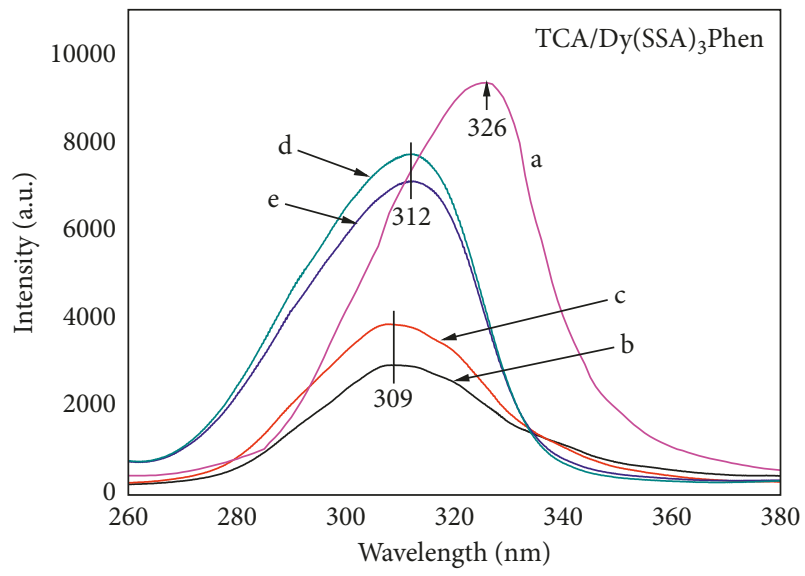

Figure 5: Excitation spectra of Dy(SSA $)_{3}$ Phen complex and TCA/Dy(SSA) ${ }_{3}$ Phen composites: a, 0/100; b, 96/4; c, 93/7; d, 90/10; e, $87 / 13$.

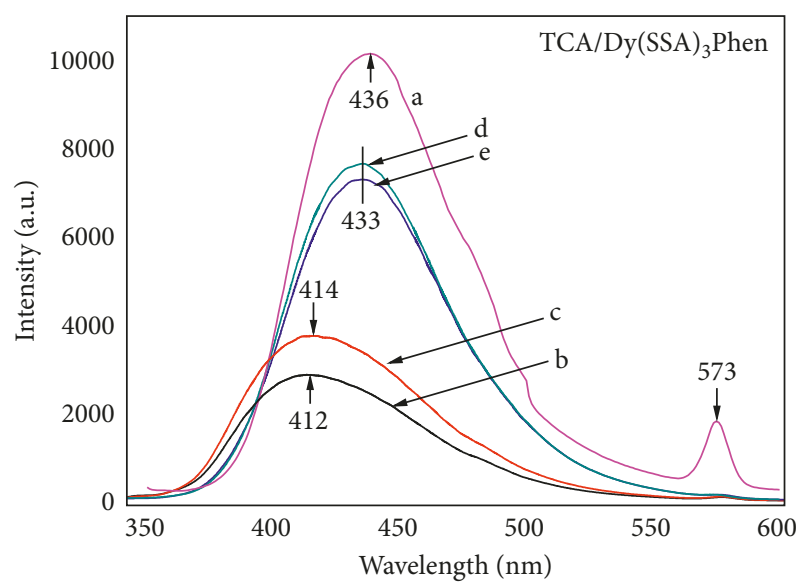

Figure 6: Emission spectra of $\mathrm{Dy}(\mathrm{SSA})_{3}$ Phen complex and TCA/Dy(SSA) ${ }_{3}$ Phen composites: a, 0/100; b, 96/4; c, 93/7; d, 90/10; e, $87 / 13$, under excitation wavelengths at $326 \mathrm{~nm}, 312 \mathrm{~nm}, 312 \mathrm{~nm}$, $309 \mathrm{~nm}$, and $309 \mathrm{~nm}$, respectively.

$573 \mathrm{~nm}$ is ascribed to the ${ }^{4} \mathrm{~F}_{9 / 2} \longrightarrow{ }^{6} \mathrm{H}_{13 / 2}$ transition of $\mathrm{Dy}^{3+}$ ions $[2,35,36]$. This is probably because the introduction of the central $\mathrm{Dy}^{3+}$ ions is propitious to the energy transfers of the SSA ligands, due to the larger planar structure of the Dy $(\mathrm{SSA})_{3}$ Phen complex (Figure 4) [36]. The Commission Internationale de L'Eclairage (CIE) coordinate of the pure complex is calculated from its emission spectrum excited at $326 \mathrm{~nm}$. The coordinates $(0.15,0.09)$ indicate that the complex emits blue light, as shown in Figure 7. Obviously, as for the TCA/Dy $(\mathrm{SSA})_{3}$ Phen composites, there are intense broad emission bands in the $360 \mathrm{~nm}-500 \mathrm{~nm}$ wavelength range in the emission spectra, with the maxima at $412 \mathrm{~nm}$, $414 \mathrm{~nm}, 434 \mathrm{~nm}$, and $434 \mathrm{~nm}$ corresponding to the $(96 / 4)$, (93/7), (90/10), and (87/13) composites, respectively, upon the optimum excitation wavelengths. This suggests that the doped Dy(SSA) ${ }_{3}$ Phen complexes in the composites can still exhibit the characteristic emission of the SSA ligands similar to that of the pure complex. Dissimilarly, the weak bands at around $573 \mathrm{~nm}$ are absent in the emission spectra of all

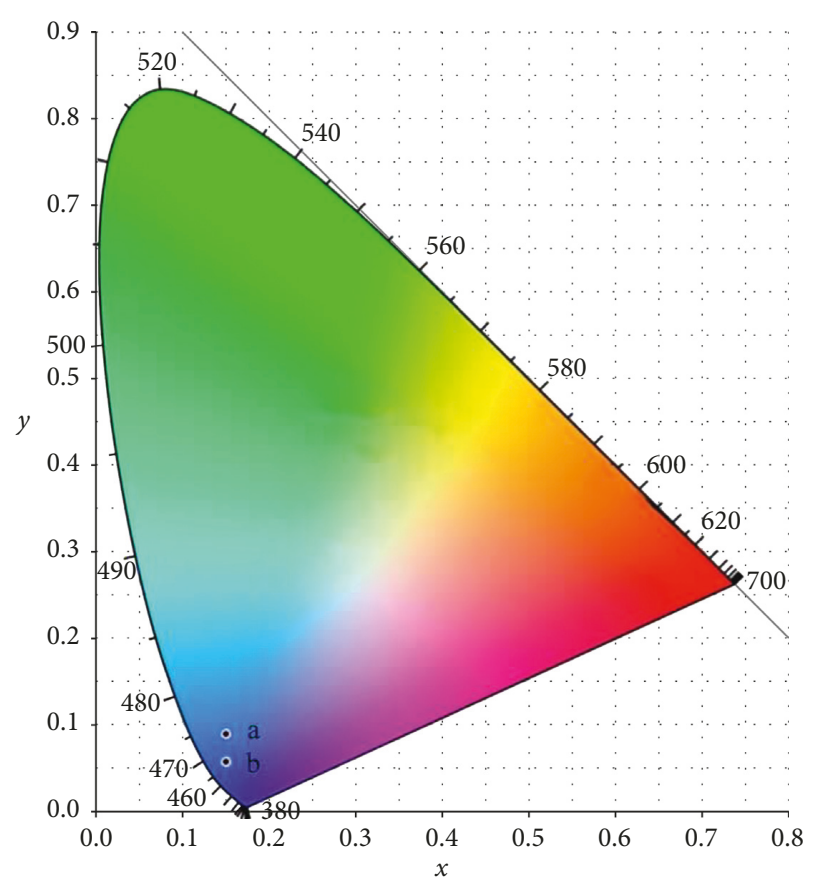

Figure 7: CIE coordinate photograph of the Dy(SSA $)_{3}$ Phen complex and the TCA/Dy(SSA) ${ }_{3}$ Phen $(95 / 5)$ composite: a, $(0.15$, $0.09)$ for the complex; $b,(0.15,0.06)$ for the composite.

composites. Furthermore, the emission spectra of the composites are also blueshifted, similar to the blueshift of the excitation spectra, which suggests that the TCA matrix has an impact on the excitation and emission processes of the additive Dy(SSA) ${ }_{3}$ Phen complex in the TCA composites.

It should be noted that a comparison of the spectral intensity of all samples indicates that the excitation and emission spectra of the pure Dy(SSA) ${ }_{3}$ Phen complex shows the strongest spectral intensity, and the spectral intensity of the TCA/Dy(SSA) ${ }_{3}$ Phen composites represents a nonlinear relationship with increasing the concentration of the added complex. When the content of $\mathrm{Dy}(\mathrm{SSA})_{3}$ Phen complex in the composites is less than $10 \mathrm{wt} . \%$, the spectral intensity of the composites gradually increases with the increase of the additive content of the complex, and the intensity of the $(87 / 13)$ composite is slightly lower than that of the $(90 / 10)$ composite. The possible reason is that in the (96/4) and (93/7) composites, the concentration of the doped complexes is relatively low so that the complexes might be completely diluted, leading to weak spectral intensity. Nevertheless, when the additive content exceeds $10 \%$, superfluous $\mathrm{Dy}(\mathrm{SSA})_{3}$ Phen complexes maybe aggregate in the TCA matrix, probably causing the concentration quenching existing in the (87/13) composite. Another possible reason is the inhomogeneity of the film. These caused the excitation, and emission intensity of the composite decreases. Clearly, when the concentration of the Dy(SSA) ${ }_{3}$ Phen complex is appropriate, namely, $10 \%$, the spectral intensity of the composite is strong and close to that of the pure complex. The coordinates $(0.15,0.06)$ indicate that the $(90 / 10)$ composite can also emit blue light, as shown in Figure 7. 
A comparison method was adopted to calculate quantum yield (QY), and quinine sulfate (in $0.1 \mathrm{M} \mathrm{H}_{2} \mathrm{SO}_{4}$ ) was the standard with literature quantum yield 0.54 at $350 \mathrm{~nm}$ [37]. The QY of the TCA/Dy(SSA) 3 Phen (90/10) composite in dimethyl sulfoxide was calculated by Equation (1), where subscripts "s" and " $\mathrm{x}$ " denote standard and test, respectively; $I$ is the integrated fluorescence intensity excited at $312 \mathrm{~nm} ; A$ is the absorption at $312 \mathrm{~nm} ; n$ is the refractive index of the solvent. The QY of the composite was calculated to be $33.5 \%$.

$$
Q Y_{\mathrm{x}}=Q Y_{\mathrm{s}}\left(\frac{I_{\mathrm{x}}}{I_{\mathrm{s}}}\right)\left(\frac{A_{\mathrm{s}}}{A_{\mathrm{x}}}\right)\left(\frac{n_{\mathrm{x}}}{n_{\mathrm{s}}}\right) \text {. }
$$

\section{Conclusion}

The Dy(SSA) ${ }_{3}$ Phen complex and the TCA/Dy(SSA) ${ }_{3}$ Phen composites all emit blue light. There are hydrogen bonds between oxygen atom of carbonyl groups from SSA and hydroxyl groups from TCA, which is favorable for the added complex to retain strong luminescence in the TCA composites. When the content of the added $\mathrm{Dy}(\mathrm{SSA})_{3}$ Phen complex is more than $10 \%$, the concentration quenching or the inhomogeneity of the film probably exists in the (87/13) composite. The TCA/Dy(SSA) ${ }_{3}$ Phen (90/10) composite possesses fine luminescent properties with the quantum yield of $33.5 \%$ and thermal stability for potential usage as blue fluorescent materials.

\section{Data Availability}

The data used to support the findings of this study are available from the corresponding author upon request.

\section{Conflicts of Interest}

The authors declare that they have no conflicts of interest to disclose in relation to the current research.

\section{Authors' Contributions}

Xiangwei Sun carried out the data analysis, wrote the draft, and revised the manuscript. Feiyue $\mathrm{Wu}$ participated in the design of the study and revised the manuscript. Yan Luo participated in the data analysis. Yuntao Li and Xiang Liu participated in the statistical analysis and revised the manuscript. Kunpeng Liu participated in the design of the study. Mengjun Huang conceived the study and revised the manuscript. All authors read and approved the final manuscript.

\section{Acknowledgments}

This work was financially supported by the Scientific and Technological Research Program of Chongqing Municipal Education Commission (No: KJZD-K201800301, KJ1711281, KJ1501128, KJ1601108, KJ1601121, and KJ1711266), Natural Science Foundation of Chongqing Municipal Science and Technology Commission (No: cstc2017jcyjAX0140, cstc2017jcyjAX0244), the Research Program of Yongchuan
District Water Authority of Chongqing Municipal (No: 201604), and the Research Program of Chongqing University of Arts and Sciences (No: R2015CH10, R2015CH12, and XSKY2016064).

\section{References}

[1] K. S. V. Krishna Rao, H. G. Liu, and Y. I. Lee, "Fluorescence spectroscopy of polymer systems doped with rare-earth metal ions and their complexes," Applied Spectroscopy Reviews, vol. 45, no. 6, pp. 409-446, 2010.

[2] N. Xu, C. Wang, W. Shi, S. P. Yan, P. Cheng, and D. Z. Liao, "Magnetic and luminescent properties of Sm, Eu, Tb, and Dy coordination polymers with 2-hydroxynicotinic acid," $E$ ropean Journal of Inorganic Chemistry, vol. 2011, no. 15, pp. 2387-2393, 2011.

[3] M. Ge, X. Guo, and Y. Yan, "Preparation and study on the structure and properties of rare-earth luminescent fiber," Textile Research Journal, vol. 82, no. 7, pp. 677-684, 2012.

[4] A. Kozłowska, M. Nakielska, J. Sarnecki et al., "Spectroscopic investigations of rare-earth materials for luminescent solar concentrators," Optica Applicata, vol. 41, no. 2, pp. 359-365, 2011.

[5] M. Flores, U. Caldiño, G. Córdoba, and R. Arroyo, "Luminescence enhancement of $\mathrm{Eu}^{3+}$-doped poly(acrylic acid) using 1,10-phenanthroline as antenna ligand," Optical Materials, vol. 27, no. 3, pp. 635-639, 2004.

[6] Y. Zhang, Q. Wang, C. Wang, X. Pei, and T. Wang, "Reversible emission color change of Eu-polymer complex induced by acetone," Journal of Macromolecular Science, Part B, vol. 51, no. 2, pp. 235-241, 2012.

[7] C. Yang, J. Xu, Y. Zhang et al., "Efficient monochromatic redlight-emitting pleds based on a series of nonconjugated Eupolymers containing a neutral terpyridyl ligand," Journal of Materials Chemistry C, vol. 1, no. 32, p. 4885, 2013.

[8] M. Bortoluzzi, G. Paolucci, M. Gatto et al., "Preparation of photoluminescent PMMA doped with tris(pyrazol-1-yl) borate lanthanide complexes," Journal of Luminescence, vol. 132, no. 9, pp. 2378-2384, 2012.

[9] Y. Mei and B. Yan, "White luminescent hybrid soft materials of lanthanide $\left(\mathrm{Eu}^{3+}, \mathrm{Sm}^{3+}\right)$ beta-diketonates and $\mathrm{Ag} / \mathrm{Ag}_{2} \mathrm{~S}$ nanoparticles based with thiol-functionalized ionic liquid bridge," Inorganic Chemistry Communications, vol. 40, pp. 39-42, 2014.

[10] H. Y. Li, J. Wu, W. Huang et al., "Synthesis and photoluminescent properties of five homodinuclear lanthanide $\left(\mathrm{Ln}^{3+}=\mathrm{Eu}^{3+}, \mathrm{Sm}^{3+}, \mathrm{Er}^{3+}, \mathrm{Yb}^{3+}, \mathrm{Pr}^{3+}\right)$ complexes," Journal of Photochemistry and Photobiology A: Chemistry, vol. 208, no. 2-3, pp. 110-116, 2009.

[11] Y. Ma and Y. Wang, "Recent advances in the sensitized luminescence of organic europium complexes," Coordination Chemistry Reviews, vol. 254, no. 9-10, pp. 972-990, 2010.

[12] X. J. Sun, W. X. Li, W. J. Chai, T. Ren, and X. Y. Shi, "The studies of enhanced fluorescence in the two novel ternary rare-earth complex systems," Journal of Fluorescence, vol. 20, no. 2, pp. 453-461, 2010.

[13] B. Gao, W. Zhang, Z. Zhang, and Q. Lei, "Preparation of polymer-rare earth complex using salicylic acid-containing polystyrene and its fluorescence emission property," Journal of Luminescence, vol. 132, no. 8, pp. 2005-2011, 2012.

[14] Z. Hong, W. L. Li, D. Zhao et al., "White light emission from OEL devices based on organic dysprosium-complex," Synthetic Metals, vol. 111-112, no. 4, pp. 43-45, 2000. 
[15] W. G. Quirino, C. Legnani, R. M. B. dos Santos et al., "Electroluminescent devices based on rare-earth tetrakis $\beta$-diketonate complexes," Thin Solid Films, vol. 517, no. 3, pp. 1096-1100, 2008.

[16] S. Biju, L.-J. Xu, M. A. H. Alvesc, R. O. Freirec, and Z.-N. Chen, "Bright orange and red light-emitting diodes of new visible light excitable tetrakis-ln- $\beta$-diketonate $\left(\mathrm{Ln}=\mathrm{Sm}^{3}\right.$ ${ }^{+}, \mathrm{Eu}^{3+}$ ) complexes," New Journal of Chemistry, vol. 41, no. 4, pp. 1473-1484, 2013.

[17] T. Cantat, F. Jaroschik, F. Nief, L. Ricard, N. Mezailles, and P. Le Floch, "New mono- and bis-carbene samarium complexes: synthesis, X-ray crystal structures and reactivity," Chemical Communications, vol. 41, no. 41, pp. 5178-5180, 2005.

[18] J. Priya, N. K. Gondia, A. K. Kunti, and S. K. Sharma, "Pure white light emitting tetrakis $\beta$-diketonate dysprosium complexes for OLED applications," ECS Journal of Solid State Science and Technology, vol. 5, no. 10, pp. R166-R171, 2016.

[19] P. P. Lima, S. A. Junior, O. L. Malta et al., "Synthesis, characterization and luminescence properties of $\mathrm{Eu}^{3+} 3$ phenyl-4-(4-toluoyl)-5-isoxazolonate based organicinorganic hybrids," European Journal of Inorganic Chemistry, vol. 2006, no. 19, pp. 3923-3929, 2006.

[20] J. G. Kang, H. G. Cho, J. G. Kim, and K. S. Choi, "Thermal and luminescent properties of polymeric acidic organophosphorus complexes of $\mathrm{Eu}(\mathrm{III})$ and $\mathrm{Tb}(\mathrm{III})$ undoped and doped with 1,10-phenanthroline," Materials Chemistry and Physics, vol. 91, no. 1, pp. 172-179, 2005.

[21] D. B. Raj, B. Francis, M. L. Reddy, R. R. Butorac, V. M. Lynch, and A. H. Cowley, "Highly luminescent poly(methyl methacrylate)-incorporated europium complex supported by a carbazole-based fluorinated beta-diketonate ligand and a 4,5-bis(diphenylphosphino)-9,9-dimethylxanthene oxide co-ligand," Inorganic Chemistry, vol. 49, no. 19, pp. 90559063, 2010.

[22] Q. F. Li, D. Yue, W. Lu, X. Zhang, C. Li, and Z. Wang, "Hybrid luminescence materials assembled by $\left[\operatorname{Ln}(\mathrm{DPA})_{3}\right]^{3-}$ and mesoporous host through ion-pairing interactions with high quantum efficiencies and long lifetimes," Scientific Reports, vol. 5 , p. 8385,2015 .

[23] J. Zhang, L. Zhang, Y. Chen, X. Huang, L. Wang, and Q. Zhang, "Influence of different carboxylic acid ligands on luminescent properties of $\mathrm{Eu}(\mathrm{Lc})_{3}$ phen $(\mathrm{Lc}=\mathrm{MAA}, \mathrm{AA}, \mathrm{BA}$, SA) complexes," Journal of Nanomaterials, vol. 2013, Article ID 768535, 8 pages, 2013

[24] T. Zhang, R. F. Li, X. Feng, S. W. Ng, and R. F. Bai, "Synthesis, crystal structure and characterization of a lanthanide complex based on 4-(4-oxypyridinium-1-yl) phthalic acid and 1,10phenanthroline," Inorganic and Nano-Metal Chemistry, vol. 47, no. 3, pp. 375-379, 2016.

[25] L. Liu, Z. Xu, Z. Lou, F. Zhang, B. Sun, and J. Pei, "Photoluminescence and electroluminescence mechanism on a novel complex $\mathrm{Eu}(\mathrm{o}-\mathrm{BBA})_{3}$ (phen)," Journal of Luminescence, vol. 122-123, pp. 961-963, 2007.

[26] X. Liu, J. Zhu, and Z. Xu, "Luminescent properties of tricellulose acetate composites doped with rare earth terbium (iii) complex," Nanomaterials and Energy, vol. 3, no. 1, pp. 25-29, 2014.

[27] A. K. Mann, D. Varandani, B. R. Mehta, L. K. Malhotra, G. Mangamma, and A. K. Tyagi, "Synthesis and luminescence properties of alumina encapsulated InN nanorods," Journal of Nanoscience and Nanotechnology, vol. 8, no. 12, pp. 62906296, 2008.
[28] X. Liu, J. Zhu, H. Ni, B. Ma, and L. Liu, "Luminescent properties of a polymer photoluminescent composite containing the binuclear $(\mathrm{Eu}, \mathrm{Tb})$ complex as an emitter," Journal of Macromolecular Science, Part B, vol. 55, no. 1, pp. 20-32, 2015.

[29] L. Yuguang, Z. Jingchang, C. Weiliang, Z. Fujun, and $\mathrm{X}$. Zheng, "Syntheses and characterization of binuclear complexes $\mathrm{Tb}_{1-\mathrm{x}} \mathrm{Eu}_{\mathrm{x}}(\mathrm{TTA})_{3}$ phen," Journal of Rare Earths, vol. 25, no. 3, pp. 296-301, 2007.

[30] T. V. Koksharova, S. V. Kurando, and I. V. Stoyanova, "Coordination compounds of 3d-metals salicylates with thiosemicarbazide," Russian Journal of General Chemistry, vol. 82, no. 9, pp. 1481-1484, 2012.

[31] C. Yang, J. Luo, J. Ma, M. Lu, L. Liang, and B. Tong, "Synthesis and photoluminescent properties of four novel trinuclear europium complexes based on two tris- $\beta$-diketones ligands," Dyes and Pigments, vol. 92, no. 1, pp. 696-704, 2012.

[32] X. W. Yang, W. J. Sun, C. Y. Ke, H. G. Zhang, X. Y. Wang, and S. L. Gao, "Thermochemical properties of rare earth complexes with salicylic acid," Thermochimica Acta, vol. 463, no. 1-2, pp. 60-64, 2007.

[33] N. Španić, "Chemical and thermal properties of cellulose acetate prepared from white willow (Salix alba) and black alder (Alnus glutinosa) as a potential polymeric base of biocomposite materials," Chemical and Biochemical Engineering Quarterly, vol. 29, no. 3, pp. 357-365, 2015.

[34] M. C. C. Lucena, A. E. V. Alencar, S. E. Mazzeto, and S. A. Soares, "The effect of additives on the thermal degradation of cellulose acetate," Polymer Degradation and Stability, vol. 80, no. 1, pp. 149-155, 2003.

[35] J. Y. Rodrigues Silva, L. Lourenco da Luz, F. G. Martinez Mauricio et al., "Lanthanide-organic gels (LOGs) as a multifunctional supramolecular smart platform," ACS Applied Materials \& Interfaces, vol. 9, no. 19, pp. 16458-16465, 2017.

[36] R. X. Wang, C. B. Liu, and G. Y. Zhu, "Study and application of a new $\mathrm{L} *-\mathrm{L}$ type fluorescence enhancement system of lanthanum-phenyl salicylate-trioctyl phosphine oxide-triton X-100," Journal of Shandong University, vol. 40, no. 2, pp. 92-95, 2005.

[37] J. E. Gill, "The fluorescence excitation spectrum of quinine bisulfate," Photochemistry and Photobiology, vol. 9, no. 4, pp. 313-322, 1969. 

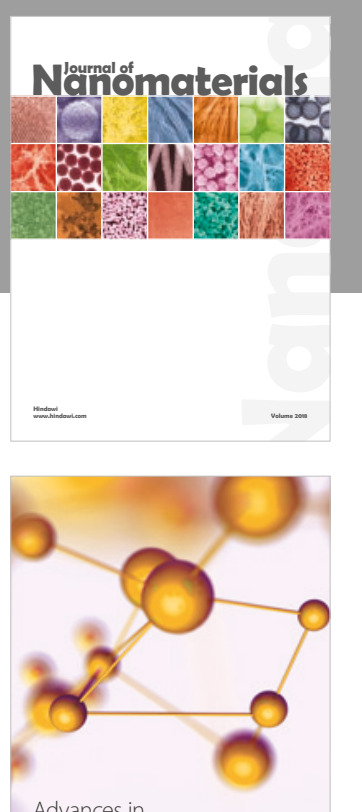

Physical Chemistry
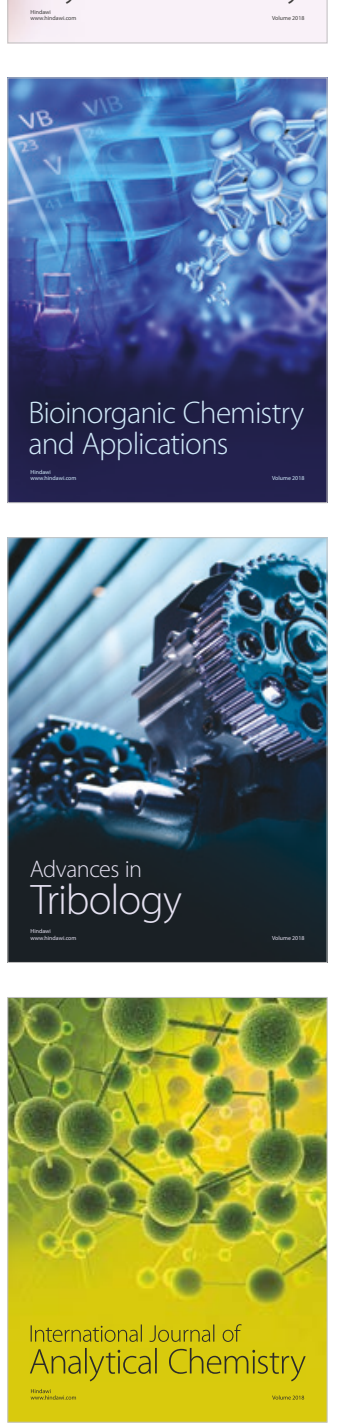

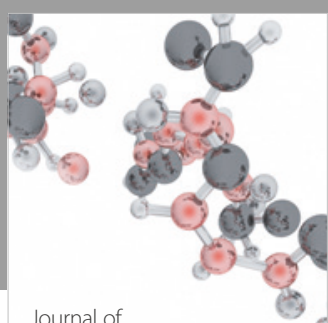

Analytical Methods

in Chemistry

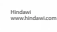

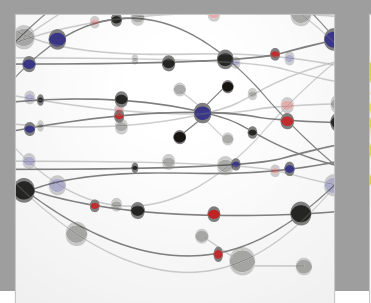

The Scientific World Journal

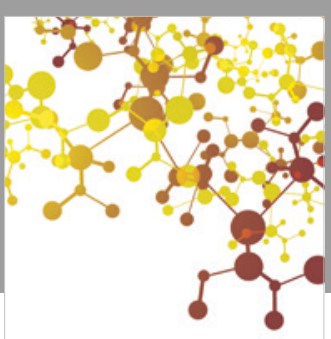

Journal of

Applied Chemistry
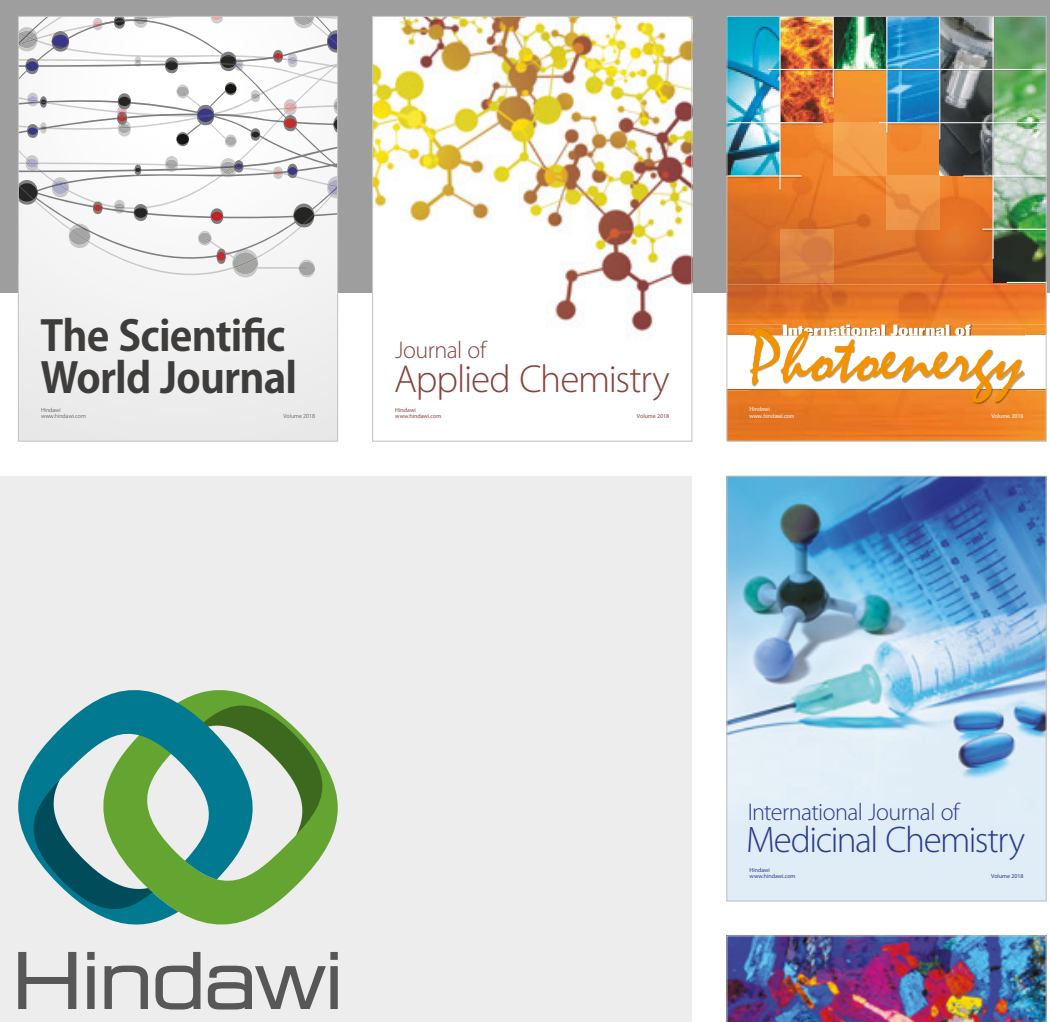

Submit your manuscripts at

www.hindawi.com
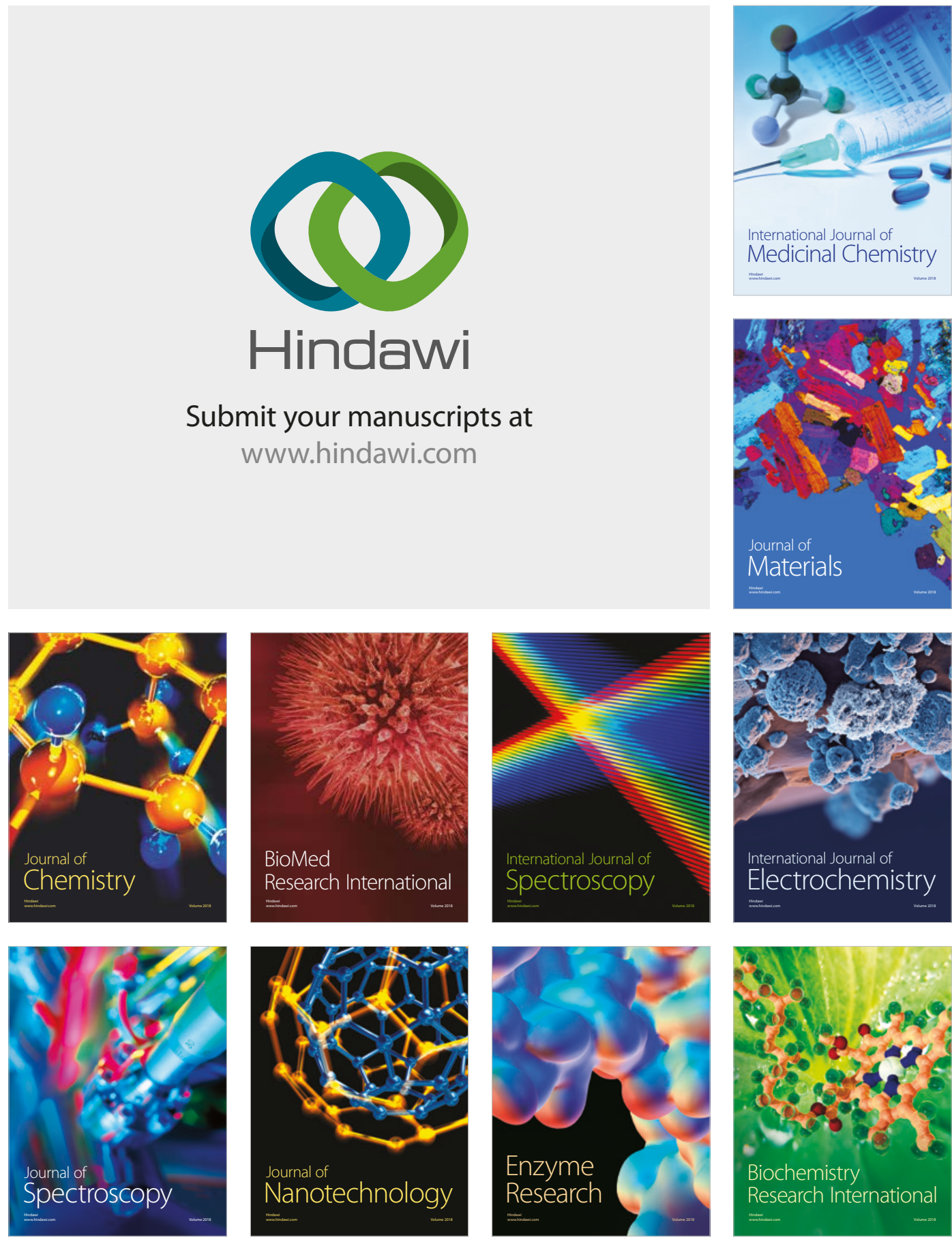
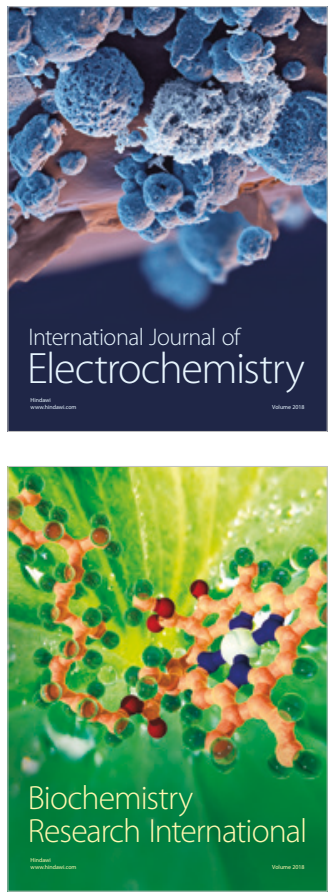